\title{
Fibrous dysplasia of maxilla - A rare case report
}

\author{
Vijaya R Kamble ${ }^{1}$, Shalini N Waghmare ${ }^{1}$, Aditi V Rangari ${ }^{1}$, Mangala Meti², Pritam Pohankar ${ }^{3}$, \\ Swati Paraye ${ }^{4}$ \\ From ${ }^{1}$ Students, ${ }^{2}$ Head of Department, ${ }^{3}$ Senior Lecture, ${ }^{4}$ Reader, Department of Oral Medicine Diagnosis and Radiology, Saraswati Dhanvantari \\ Dental College and Hospital, Parbhani, Maharashtra, India
}

\begin{abstract}
Fibrous dysplasia (FD) is a skeletal developmental anomaly, which is non-hereditary in origin and idiopathic in nature. It is a benign fibro-osseous disease that affects one or more bones. It usually affects unilaterally and is seen in the posterior region. Approximately $0.5 \%$ of untreated cases show the malignant transformation. We report the case of a 35-year-old female patient with FD involving the maxilla. The clinical diagnostic approach, different imaging modalities, and histological examination methods for definitive diagnosis have been elaborated.
\end{abstract}

Key words: Fibrous dysplasia, Jaffe-Lichtenstein, McCune-Albright's disease, Monostotic, Polyostotic

$\mathrm{F}$ ibrous dysplasia (FD) is classified as a benign fibro-osseous lesion that occurs due to a disturbance of bone metabolism. The newly formed abnormal bone containing fibrous connective tissue replaces the normal bone. FD was originally described by Lichtenstein in 1938 and by Lichtenstein and Jaffe in 1942 [1]. FD is a type of hamartoma, where the normal medullary bone is replaced by poorly calcified and immature bone $[2,3]$.

The most common symptom seen during childhood and adolescence is bone pain and repeated fractures followed by bine deformity and neurological compression, especially seen with facial bone and skull involvement [4]. The commonly involved skeletal sites include long bines, craniofacial bones, ribs, and pelvis [5].

Here, we report a case of FD involving maxilla in a female patient who was diagnosed with the help of various imaging modalities and confirmed the diagnosis with an incisional biopsy.

\section{CASE REPORT}

A 35-year-old female patient reported to the department of oral medicine and radiology with the chief complaint of a slowly growing painless swelling in the left upper back teeth region of the jaw for 1 year. There was no significant extra-oral finding (Fig. 1a). Intraorally, the patient gives a history of a swelling which initially was pea sized but gradually, increased to attain the present size over a period of time. There was no relevant history

\section{Access this article online}

Received - 10 October 2021

Initial Review - 29 October 2021

Accepted - 02 November 2021

DOI: $10.32677 /$ ijcr.v7i11.3108 of trauma, paresthesia, and difficulty in chewing food, and it was not associated with any other symptoms.

On examination, the patient was well oriented to time, place, and person. The swelling measuring approximately $5 \times 4 \mathrm{~cm}$ was present in the left maxillary alveolar bone region extending from 23 to 28 with buccal and palatal cortical plate expansion. The obliteration of the buccal vestibule was also noted (Fig. 1b). Mucosa overlying the swelling appeared to be normal. All the inspectory findings were confirmed on palpation. The consistency of the swelling was bony hard and non-tender. No local rise in temperature was noted. The teeth in this region were vital. Considering all the inspectory and palpatory findings along with the history, a provisional diagnosis of fibro-osseous lesion affecting the left maxilla was made.

Complete hemogram showed all the parameters to be within normal limits. Serological investigations including serum calcium, serum phosphorus, and alkaline phosphatase were also within normal limits.

The radiographic examination included a maxillary cross-sectional occlusal radiograph, orthopantomagram (OPG), and facial computed tomography (CT) scan. The maxillary cross-sectional occlusal radiograph revealed a homogenous, granular radio-opacity involving the left maxillary alveolar bone with the expansion of the buccal cortical plate which extends from 23 to 27. A typical granular or ground-glass appearance can be noted. The margins of the lesions blended with the adjacent normal areas of the bone (Fig. 2a). OPG revealed homogeneous granular radio-opacity causing obliteration of the maxillary sinus (Fig. 2b). Facial CT shows a diffuse, expansile lesion with uniform increased attenuation (ground-glass appearance)

Correspondence to: Dr. Vijaya R Kamble, "Shivkrupa," Samadhan Colony, Near Deshmukh Hotel, Karegaon Road, Parbhani, Maharashtra, India. E-mail: vjaya.kamble@gmail.com

(C) 2021 Creative Commons Attribution-NonCommercial 4.0 International License (CC BY-NC-ND 4.0). 
which involves the maxillary alveolar process in relation to the left canine and molar teeth, floor, anterior, and the lateral walls of the maxillary sinus (Fig. 2c and d). The radiographic diagnosis was made as FD involving the left maxilla.

The lesion was surgically excised and sent for histopathological examination. The excised tissue showed a benign fibro-osseous lesion formed by a hypercellular fibroblastic stroma consisting of spindle cells arranged in whorls in a storiform pattern and also in loose sheets with intervening collagen. Among these arrangements, there were immature woven bones with Chinese letter configuration, thus all the features were highly suggestive of FD. The overall features were highly suggestive of FD (Fig. 3).

\section{DISCUSSION}

FD represents $2.5 \%$ of all bone tumors and $7 \%$ of all benign bone tumors, which occur due to congenital, metabolic, and genetic disturbances [6]. It is manifested in the first decade of life in approximately $70 \%$ of the cases and presents with slow growth, stabilizes at puberty, along with the skeleton. In the above-mentioned case, the patient is in the fourth decade of life which is rare. FD is frequently seen in females, with a $37 \%$ recurrence in adults [7].

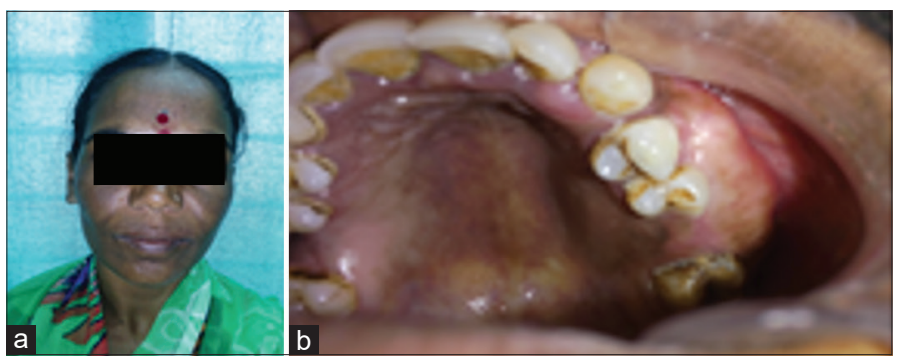

Figure 1: (a) Extraoral examination showed no significant findings; (b) intraoral swelling present in the left maxillary alveolar bone region extending from 23 to 28 with buccal and palatal cortical plate expansion

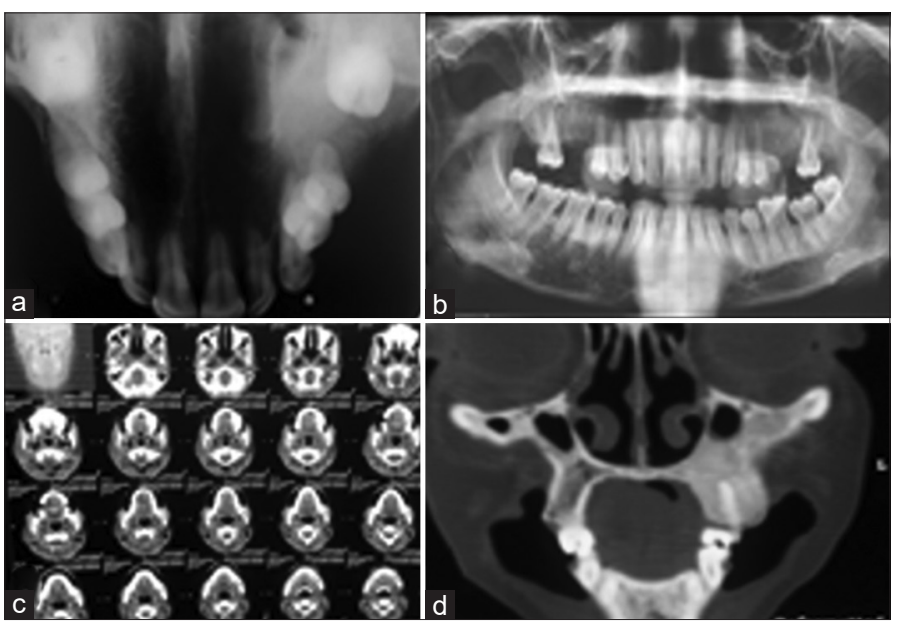

Figure 2: (a) Occlusal radiograph ground-glass appearance; (b) orthopantomagram revealed homogeneous granular radioopacity causing obliteration of the maxillary sinus; (c and d) facial computed tomography shows a diffuse, expansile lesion with uniform increased attenuation (ground-glass appearance) which involves the maxillary alveolar process in relation to the left canine and molar teeth, floor, anterior, and the lateral walls of maxillary sinus
FD can be divided into three categories: Monostotic (74\%), polyostotic (13\%), and craniofacial (13\%) [8]. Monostotic affects only one bone. It is the most common type, has no gender prediction, and is found in $70 \%$ of all involved cases, with most commonly affecting the gnathic bones [9]. Polyostotic type of FD is an uncommon type, having a higher prevalence for females. Few to $75 \%$ of the entire skeleton can be affected in polyostotic $\mathrm{FD}$, with craniofacial involvement and shows a predilection for one side of the body [10]. The polyostotic form affects multiple bones and can be further divided into three subtypes: Craniofacial, which involves the bones of the craniofacial region also including mandible and maxilla; Lichtenstein-Jaffe type, which shows the involvement of multiple bones of the skeleton along with brown pigmentations of the skin; and Albright syndrome, which shows the involvement of several skeletal bones, brown-to-brown pigmentation on the skin along with endocrine impairment, which is markedly seen in early puberty affecting girls. Polyostotic form accounts for $20-30 \%$ of the cases [11].

The exact etiologic cause of FD is not known. However, it has been classified as a developmental origin according to the World Health Organization (WHO). The condition is not hereditary in origin. Many articles and researchers have linked its etiologic factor as the mutation in the Gs $\alpha$ gene located at chromosome 20q13.2. The mutation occurs specifically at position 201, which is occupied by arginine (R201) and further replaced by either cysteine (R201C) or histidine (R201H) [12]. The bone is actually replaced by fibrous tissue in FD [13]. The following flowchart depicts the pathogenesis of FD (Fig. 4) [14].

Clinically, it presents as an asymptomatic, unilateral, and slowly progressive enlargement of the involved bone or jaw. Involvement of buccal cortical plate presents as a fusiform swelling that causes displacement of teeth leading to malocclusion [15]. In FD, the clinical finding and radiographic features are very specific, which help the practitioner to diagnose without biopsy.

There are several variations seen density and trabecular pattern of FD lesions. Early lesions of FD tend to show more radiolucency than the mature lesions and, in rare cases, it may show granular internal septa, giving it a multilocular appearance. The trabeculae in the affected bones are shorter, thinner, irregularly shaped, and more numerous than the normal trabeculae which lead to a variable

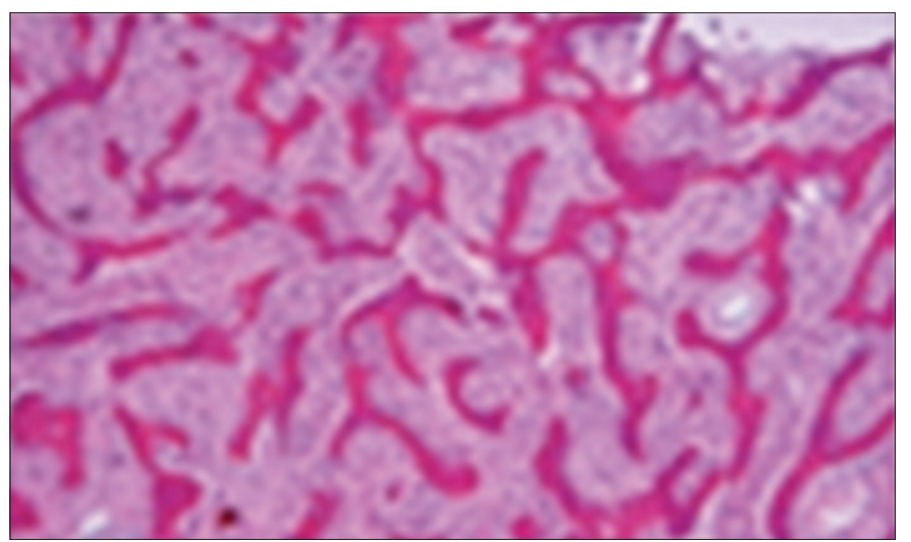

Figure 3: Histopathological examination showing spindle cells arranged in whorls in storiform pattern and also in loose sheets with intervening collagen 


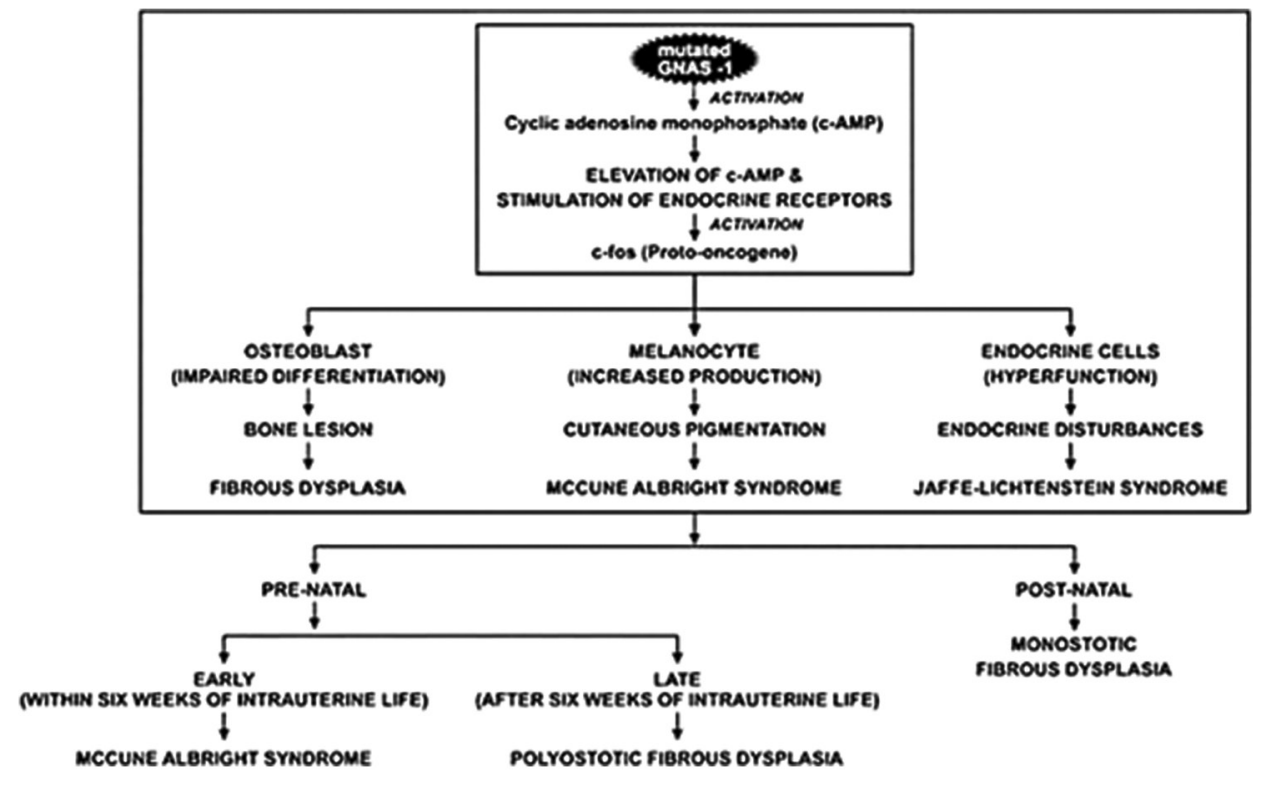

Figure 4: Flowchart of pathogenesis of fibrous dysplasia

radio-opaque pattern. Apart from this, various appearances can be seen such as granular appearance ("ground-glass" appearance, which resembles small fragments of a shattered windshield), Peau d'orange (which resembles the surface of an orange), a wispy arrangement (also known as cotton wool appearance), or an amorphous, dense pattern. Another typically seen is the fingerprint pattern, which occurs due to the organization of the abnormal trabeculae into a swirling pattern [16].

The differential diagnosis based on radiographic similarities includes ameloblastoma, ameloblastic fibroma, ameloblastic odontoma, ameloblastic fibro-odontoma, central giant cell granuloma, odontogenic cyst, ossifying fibroma, osseous dysplasia, chronic sclerosing osteomyelitis, and osteosarcoma, should be considered. All of these can be differentiated based on clinical and histopathological findings.

Clinical management of FD is quite complex based on the age of the patient. The treatment protocol for FD includes observation, medical treatment, and surgery. FD lesions have a high risk of pathologic fracture or deformity which requires proper clinical observation. There is no specific medication capable of altering the disease process, but medical management can be given for palliation; which involves bisphosphonates, which shows improvement of function, pain relief, and lower fracture risk. In histopathologically confirmed patients, surgery is carried out for deformity correction, prevention of pathologic fracture, and/or elimination of symptomatic lesions. The standard care management involves conservative treatment, with the removal of diseased bone through an intraoral approach. Bone grafts used are cortical bone grafts as it has an excellent quality of remodeled cortical bone, compared to cancellous bone grafts or bone graft substitute [5].

\section{CONCLUSION}

A single confirmed case of FD affecting either the maxilla or mandible is rare. It becomes quite difficult to differentiate these benign bone disorders from malignant ones. To establish a diagnosis of FD, adequate patient history, thorough examination, and radiographic assessment are often sufficient. The treatment protocol depends on the age of the patient, facial involvement, presence or absence of facial asymmetry, and future rehabilitation. It is essential to surgically remove most of the part of tissue without causing any disfigurement, functional loss, or loss of function of noble structures. Any significant deformity, tremendous pain, or pathological fracture may indicate the need for surgical intervention. A proper and regular follow-up is a must, to detect relapse or any malignant changes at an early stage.

\section{REFERENCES}

1. Mahadesh J, Gowda C, Devi L, Kokila G. Fibrous dysplasia of the jaw bones: Clinical, radiographical and histopathological features. Report of two cases. J Dent Sci Res 2011;2:18-25.

2. Ben Hadj Hamida F, Jlaiel R, Ben Rayana N, Mahjoub H, Mellouli T, Ghorbel M, et al. Craniofacial fibrous dysplasia: A case report. J Fr Ophtalmol 2005;28:e6.

3. Ozek C, Gundogan H, Bilkay U, Tokat C, Gurler T, Songur E. Craniomaxillofacial fibrous dysplasia. J Craniofac Surg 2002;13:382-9.

4. Coley B. Neoplasms of Bone and Related Conditions. Vol. 1. New York, USA: Paul Hocher Inc.; 1960.

5. DiCaprio MR, Enneking WF. Fibrous dysplasia. Pathophysiology, evaluation, and treatment. J Bone Joint Surg Am 2005;87:1848-64.

6. Edgerton MT, Persing JA, Jane JA. The surgical treatment of fibrous dysplasia. With emphasis on recent contributions from cranio-maxillo-facial surgery. Ann Surg 1985;202:459-79.

7. Jan M, Dweik A, Destrieux C, Djebbari Y. Fronto-orbital sphenoidal fibrous dysplasia. Neurosurgery 1994;34:544-7.

8. Eversole LR, Sabes WR, Rovin S. Fibrous dysplasia: A nosologic problem in the diagnosis of fibro-osseous lesions of the jaws. J Oral Pathol 1972;1:189-220.

9. Saueressig F, Oliveira MG. Displasia fibrosa poliostótica associada à síndrome de Mmccune-albright: Relato de caso. Rev Bras Patol Oral 2004;3:70-6.

10. Botelho RA, Tornin OS, Yamashiro I, Menezes MC, Furlan S, Ridelenski M, et al. Características tomográficas da dysplasia fibrosa craniofacial: Estudo retrospectivo de 14 casos. Radiol Bras 2006;39:269-72.

11. Pontual ML, Tuji FM, Yoo HJ, Bóscolo FN, Almeida SM. Estudo 
epidemiológico da displasia fibrosa dos maxilares numa amostra da população brasileira. Odontol Clin Científ 2004;3:25-30.

12. Agarwal MM, Balaji N, Sumathi MK, Sunitha JD, Dawar G, Rallan NS. Fibrous dysplasia: A review. TMU J Dent 2014;1:25-9.

13. Cholakova R, Kanasirska P, Kanasirski N, Chenchev I, Dinkova A. Fibrous dysplasia in the maxillomandibular region-case report. JIMAB 2010;16:10-3.

14. Srichinthu K, Yoithapprabhunath T, Chitturi RT, Yamunadevi A, Potsangbam AD, Singh DN. Fibro osseous lesions-classifications, pathophysiology and importance of radiology: A short review. Int Biol Biomed J 2016;2:11-20.

15. Ganapathy N. Fibrous dysplasia of jaw: A case report and update on pathogenesis. JIADS 2011;2:54-6.

16. Canitezer G, Gunduz K, Ozden B, Kose HI. Monostotic fibrous dysplasia: A case report. Dentistry 2012;3:1-4.

Funding: None; Conflicts of Interest: None Stated.

How to cite this article: Kamble VR, Waghmare SN, Rangari AV, Meti M, Pohankar P, Paraye S. Fibrous dysplasia of maxilla - A rare case report. Indian J Case Reports. 2021;7(11):485-488. 